\title{
Modelling Non-coaxiality and Strain Localisation in Sand: the Role of Fabric and Its Evolution
}

\author{
Jidong Zhao \\ Department of Civil and Environmental Engineering, Hong Kong University of Science and Technology \\ Clearwater Bay, Kowloon, Hong Kong \\ Zhiwei Gao \\ School of Engineering, University of Glasgow, Oakfield Avenue, Glasgow, G12 8LT, UK
}

\begin{abstract}
We present a critical state sand plasticity model accounting for the effect of fabric and its evolution, and apply it to modelling the non-coaxial behaviour and shear localisation phenomenon in sand. The model is developed within the framework of Anisotropic Critical State Theory. A novel fabric evolution law is further proposed to guide the fabric anisotropy evolving towards a unique critical state under continuous shear. The yield surface, hardening law and dilatancy relation of the model feature an explicit dependence on the fabric anisotropy, and are hence affected by its evolution during the deformation of sand. The model formulation leads naturally to a non-coaxial flow rule which enables it to capture the non-coaxial behaviour in sand with ease. We have further applied the model to the prediction of shear localisation in sand. We identify two competing mechanisms dictating the occurrence and further development of shear band in sand, one attributable to the evolution of fabric and the other imposed by the boundary constraints. The study helps to shed light on the fundamental understanding of sand behaviour such as non-coaxility and the phenomenon of strain localisation.
\end{abstract}

\section{INTRODUCTION}

Soil fabric affects important soil behaviour including strength, dilatancy and critical state. Fabric anisotropy in sand changes constantly with the strain to establish a compatible internal structure with the applied stress and exhibits unique characteristics at critical state (Zhao \& Guo 2013, Guo \& Zhao 2013). Accurate modelling of sand behaviour needs to fully consider the evolving nature of fabric during the loading course. The majority of existing studies on fabric anisotropy have either considered a constant fabric tensor in the model formulation, or have been based on the rotation of yield surface. Yield surface rotation cannot adequately account for the anisotropic nature of sand related to particle orientation, contact normal and void space distribution, since the direction and magnitude of rotation are associated with the initial stress state but not these physical properties of soil. Despite the convenience and simplicity it may offer, the employment of a constant fabric tensor ignores the evolution of fabric anisotropy during the deformation of the soil, which gives rise to inconsistent predictions with experimental and numerical observations. It may also lead to non-uniqueness of critical state line.

This paper presents a general three-dimensional critical state sand plasticity model with full account of the effect of fabric and its evolution recently developed by the authors (Gao et al. 2014). The model has been developed within the Anisotropic Critical State Theory proposed by Li \& Dafalias (2012). It features an explicit dependence of the yield surface, the hardening law and the dilatancy relation on fabric anisotropy. With a novel fabric evolution law being proposed, all these are further made dependent on the evolution of fabric. The model formulation naturally leads to a non-coaxial flow rule. It shows excellent predictive capacity in reproducing the typical sand behaviour observed in laboratory tests, and provides convincing physical explanations on such phenomena as non-coaxiality.

In addition to characterisation of material behaviour in sand, we have applied the model to simulating boundary value problems. Widely regarded as an important precursor to catastrophic failures such as landslides and debris flow, strain localisation in sand has received much attention in the research community of geomechanics. The occurrence and development of strain localisation has to be closely with important microstructure such as soil fabric and fabric evolution. While the influence of initial fabric anisotropy on strain localisation has been treated be- 
fore, the correlation between fabric evolution and strain localisation remains an untouched topic. We have implemented the newly developed sand plasticity model considering fabric evolution in a displacement finite element code and have applied it to predicting the shear localisation in sand under plane strain compression. Based on the simulation of the such a well-designed boundary value problem, the interaction between fabric evolution and shear localisation in sand is investigated.

\section{SAND PLASTICITY MODEL ACCOUNTING FOR FABRIC EVOLUTION}

\subsection{Model formulation}

\subsubsection{Elastic relations}

The influence of fabric anisotropy on the elastic behaviour of sand is neglected here. The following isotropic pressure-sensitive elastic relations are employed

$$
G=G_{0} \frac{(2.97-e)^{2}}{1+e} \sqrt{p p_{a}}, \quad K=G \frac{2(1+\nu)}{3(1-2 \nu)}
$$

where $G$ and $K$ are respectively the elastic shear and bulk modulus. $G_{0}$ is a material constant. $e$ is the void ratio and $\nu$ is the Poisson's ratio (taken as a constant). $p=\sigma_{i i} / 3$ and $\sigma_{i j}$ is the stress tensor. $p_{a}$ denotes the atmospherical pressure. The following incremental elastic relations are employed to describe the nonlinear elasticity for sand:

$$
d e_{i j}^{e}=\frac{d s_{i j}}{2 G}, \quad d \epsilon_{v}^{e}=\frac{d p}{K}
$$

where $s_{i j}=$ deviator stress; $e_{i j}^{e}=$ deviator elastic strain; $\epsilon_{v}^{e}=$ volumetric elastic strain.

\subsubsection{Yield function}

To account for the influence of fabric anisotropy, the following fabric-dependent yield function is employed in the model (Gao et al. 2014):

$f=\frac{R}{g(\theta)}-H e^{-k_{h}(A-1)^{2}}=0$

where the first term $R / g(\theta)$ is a normalised shear stress, while the second term denotes a generalized shear resistance involving the influence of fabric anisotropy through $A . R=\sqrt{3 r_{i j} r_{i j} / 2}$ is the stress ratio tensor $\left(r_{i j}=\left(\sigma_{i j}-p \delta_{i j}\right) / p=s_{i j} / p\right.$; $\delta_{i j}=$ Kronecker delta); $H$ is a hardening parameter whose evolution law depends on the stress as well as internal variables including the density and fabric; $k_{h}$ is a non-negative model constant. When $k_{h}=$ 0 , the yield function degenerates to a conventional isotropic yield surface in the stress space; $g(\theta)$ is an interpolation function dependent on the Lode angel $\theta$ of $r_{i j}$ viz: $g(\theta)=\frac{\sqrt{\left(1+c^{2}\right)^{2}+4 c\left(1-c^{2}\right) \sin 3 \theta}-\left(1+c^{2}\right)}{2(1-c) \sin 3 \theta}$ where $c=M_{e} / M_{c}$ denotes the ratio between the critical state stress ratio in triaxial extension $M_{e}$ and that in triaxial compression $M_{c}$. Serving as an important component, $A$ in Eq. (3) denotes a fabric anisotropy variable defined by the first joint invariant of the fabric tensor $F_{i j}$ and the loading direction tensor $n_{i j}$ :

$A=F_{i j} n_{i j}$

where $F_{i j}$ is is a symmetric traceless fabric tensor whose norm $F=\sqrt{F_{i j} F_{i j}}$ represents the degree of fabric anisotropy. A normalised fabric tensor $F_{i j}$ is used such that $F$ is unity at critical state. The deviatoric unit loading direction tensor $n_{i j}$ is defined by

$n_{i j}=\frac{N_{i j}-N_{m n} \delta_{m n} \delta_{i j} / 3}{\left\|N_{i j}-N_{m n} \delta_{m n} \delta_{i j} / 3\right\|}$

where

$N_{i j}=\frac{\partial \bar{f}}{\partial R} \frac{\partial R}{\partial r_{i j}}-\frac{\partial \bar{f}}{\partial g(\theta)} \frac{\partial g(\theta)}{\partial \theta} \frac{\partial \theta}{\partial r_{i j}}$

where $\bar{f}=R / g(\theta)$. At critical state, $n_{i j}$ and $F_{i j}$ become co-directional (Li \& Dafalias 2012), $F$ reaches a critical state value of 1 , which results in $A=1$. In this case, the yield function in Eq. (3) becomes an isotropic critical state failure surface again. An isotropic critical state failure surface does not necessarily mean the critical state fabric is isotropic, which has been confirmed by DEM simulations (Zhao \& Guo 2013, Guo \& Zhao 2013).

\subsubsection{Fabric evolution, plastic hardening law and dilatancy relation}

The following dilatancy relation is followed:

$D=\frac{d_{1}}{M_{c} g(\theta)}\left[1+\frac{R}{M_{c} g(\theta)}\right]\left[M_{c} g(\theta) e^{m \zeta}-R\right]$

where $d_{1}$ and $m$ are two model constants. $\zeta$ is the diltatancy state parameter defined by $\mathrm{Li}$ and Dafalias (2012)

$\zeta=\psi-e_{A}(A-1)$

where $e_{A}$ is a model parameter. $\psi=e-e_{c}$ is the state parameter defined by Been and Jefferies (1985).

The following fabric evolution law is proposed:

$d F_{i j}=\langle\lambda\rangle \Theta_{i j}=\langle\lambda\rangle k_{f}\left(n_{i j}-F_{i j}\right)$

where $\lambda$ is the plastic multiplier. $k_{f}$ is a positive model constant representing the rate of fabric evolution. The fabric evolves with the plastic deformation according to Eq. (9), which renders the fabric tensor tending towards coaxiality with the loading direction $n_{i j}$. The following hardening law is employed:

$d H=\langle\lambda\rangle r_{n}=\langle\lambda\rangle \frac{G\left(1-c_{h} e\right)}{p R}\left[M_{c} g(\theta) e^{-n \zeta}-R\right](10)$

where $c_{h}$ and $n$ are two positive model parameters. 


\subsubsection{Non-coaxial associated flow rule in the deviatoric space}

The yield function in Eq. (3) includes the joint invariant $A$, which naturally produces non-coaxial deformation for associated flow rule. This is demonstrated as follows. By assuming an associated flow rule in the deviatoric stress space, the plastic strain rate will be dependent on the derives of the yield function with respect to the stress tensors. Based on Eq. (3) one can easily obtain:

$$
\frac{\partial f}{\partial r_{i j}}=N_{i j}+\frac{\partial f}{\partial A} F_{k l} \frac{\partial n_{k l}}{\partial r_{i j}}
$$

where

$N_{i j}=\frac{\partial f}{\partial R} \frac{\partial R}{\partial r_{i j}}+\frac{\partial f}{\partial g(\theta)} \frac{\partial g(\theta)}{\partial \theta} \frac{\partial \theta}{\partial r_{i j}}$

It is readily seen that the inclusion of fabric anisotropy viz $A$ in the yield function leads to $\partial f / \partial r_{i j}$ consisting of two parts. The first part $N_{i j}$ is apparently coaxial with the direction of the stress ratio $r_{i j}$ (or equivalently to the direction of the stress $\sigma_{i j}$. The second part involves fabric anisotropy $F_{i j}$ which is generally non-coaxial with $r_{i j}$ as long as fabric anisotropy is non-zero. This naturally addresses the non-coaxiality in soil modelling. Granular media generally exhibit an appreciable amount of non-coaxial deformation with the stress during early stage of shearing, which may gradually diminish with the shearing to high strain level. This can indeed be reasonably explained by the second part of Eq. (11).

\subsubsection{Incremental stress-strain relation}

Based on the above formulations, the following incremental stress strain relation can be determined based on the yield function and the elasticity relation

$d \sigma_{i j}=D_{i j k l} d \epsilon_{k l}$

where the elastoplastic stiffness tensor is

$D_{i j k l}=C_{i j k l}-h(\lambda)\left(2 G m_{i j}+\sqrt{2 / 3} K D \delta_{i j}\right) \Pi_{k l}$

where $C_{i j k l}=K \delta_{i j} \delta_{k l}+2 G\left(\delta_{i k} \delta_{j l}-\frac{1}{3} \delta_{i j} \delta_{k l}\right) . D=\frac{d \epsilon_{v}^{p}}{d \epsilon_{q}^{p}}$ is the dilatancy. $h(\lambda)$ is the Heaviside step function where $h(\lambda>0)=1$ and $h(\lambda \leq 0)=0 . \Pi_{k l}$ can be expressed by the deviator unit loading direction tensor $N_{i j}$, the derivatives based on the consistency condition of yield function and the plastic modulus $K_{p}$. For detail of the expression please refer to Gao et al. (2014).

\subsection{Model Calibration and verification}

The model has been carefully calibrated based on experimental data on dry-deposited Toyoura sand reported by Yoshimine et al. (1998). Figure 1 presents a comparison of stress-strain relations and stress path between the experimental results and model prediction for an undrained test, which shows a good coincidence.
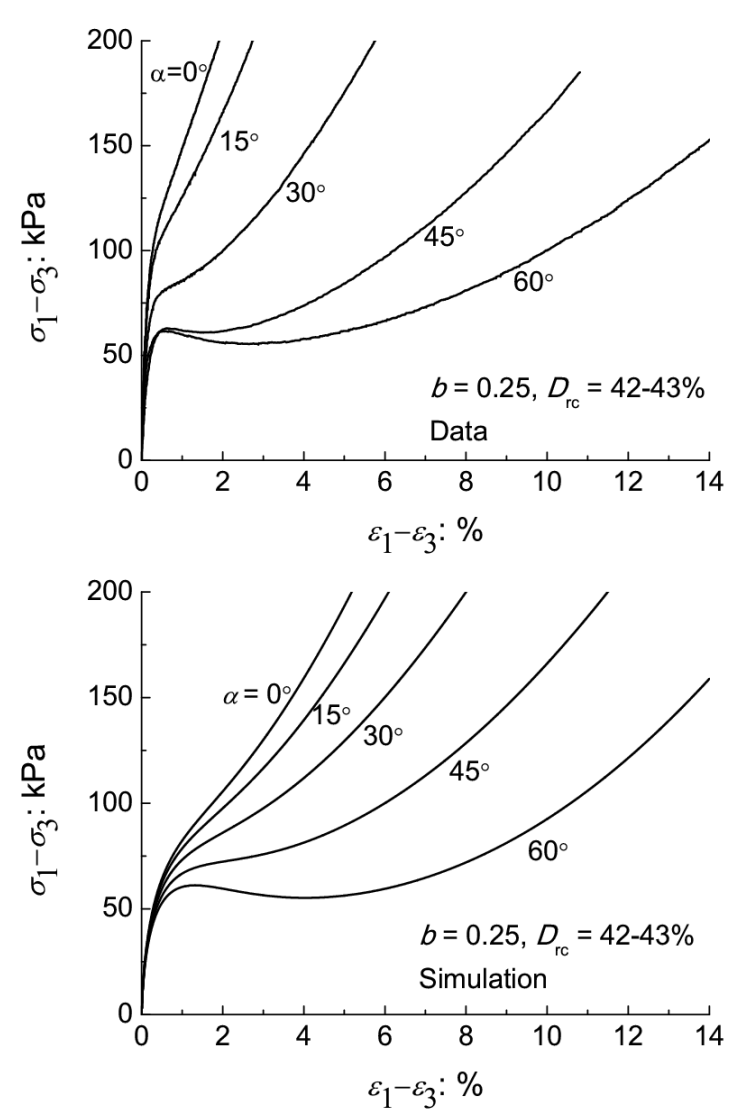

(a) Stress strain relation
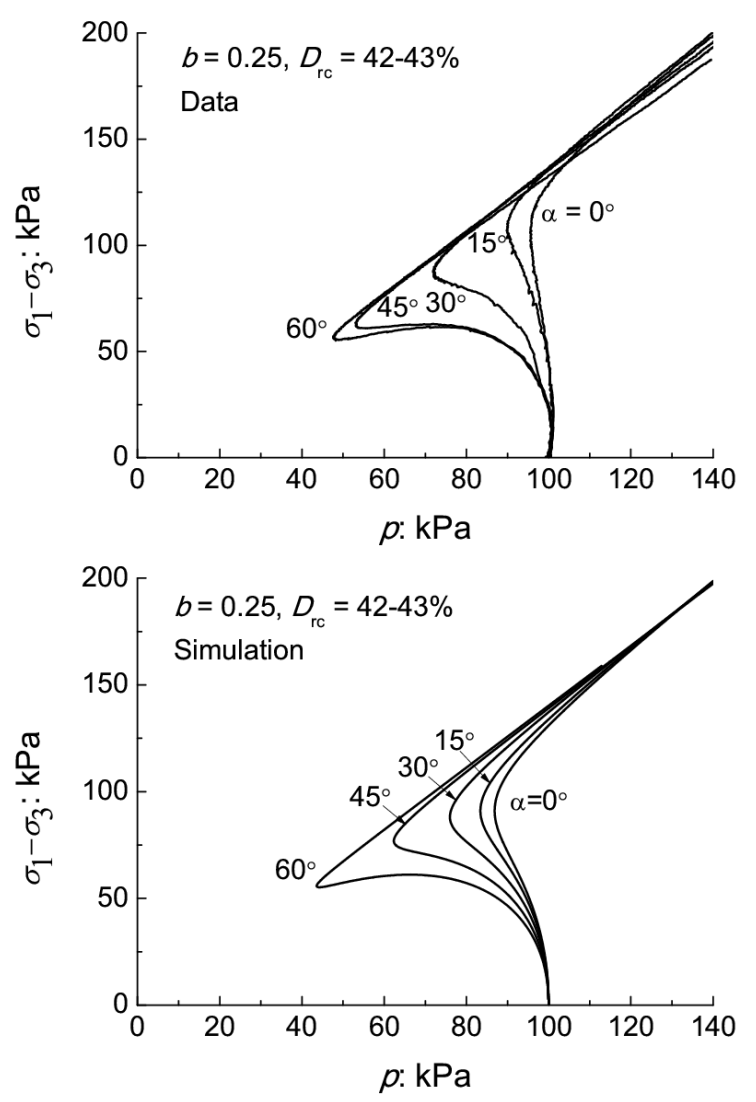

(b) Stress path

Figure 1: A comparison of model simulations and test data of the undrained behavior of dry-deposited Toyoura sand under different principal stress angle. 


\section{PREDICTING THE NON-COAXIAL BEHAVIOUR IN SAND}

As mentioned before, the proposed model features a natural non-coaxial flow rule in Eq. (11) by including an evolving fabric tensor into the yield function. This flow rule can facilitate the explanation of non-coaxial behaviour in sand. Indeed, according to Eq. (11), the plastic strain increment involves a component that does not align with the stress direction (the second term of the right-hand side of the equation), and consequently, the phenomenon of non-coaxiality may be easily handled. The non-coaxial behaviour can be caused by two cases: (a) the soil fabric and the loading direction are initially not coaxial to each other; (b) the changes of stresses and the soil fabric are not synchronised. A typical example of case (b) can be found in a typical rotational shearing test where the fabric tends to align its major principal axes to be coaxial with that of the loading direction, in an attempt to reach an optimum internal rearrangement to bear the load. The change of fabric is however always lagging behind the stress change due to its passive nature, which leads to non-coaxial stress and fabric tensor. Figure 2 demonstrates an example of the model prediction of noncoaxial behaviour for Toyoura sand under undrained shear. In the figure, $\alpha(\sigma)$ is defined by the relative angle between the direction of the major principal stress $\sigma_{1}$ and the vertical direction, while $\alpha(\epsilon)$ is defined by the relative angle between the major principal strain $\epsilon_{1}$ and the vertical direction. The difference between the two angles, $\alpha(\epsilon)-\alpha(\sigma)$, serves as a measure of noncoaxiality in sand and its variation with shear strain in presented in Figure 2. There is a good qualitative coincidence between the model simulations and the experimental data.

We further note that there is only change of principal values of fabric tensor during the development of plastic strain when $\alpha=0^{\circ}$ and $\alpha=90^{\circ}$. The direction of the fabric will align with the stress direction during the loading course for the two cases. The predicted sand response is thus coaxial, which is consistent with Gutierrez \& Ishihara (2000). In all the other cases, distinct different between $\alpha(\epsilon)$ and $\alpha(\sigma)$ in the order of average 4 to 5 degrees is found, indicting a clear evidence of non-coaxiality. The difference becomes smaller after the peak when the fabric tends to rotate towards the direction of stress. The non-coaxial behaviour is expected to entirely disappear at very large strain level. It is also observed that the with $b=0$ the test data show the $\alpha=30^{\circ}$ cases gives the maximum difference, while our model prediction points to the case of $\alpha=45^{\circ}$. The possible reason may be that sand fabric in the tested samples is not rigorously cross-anisotropic at the initial state. In general, the current model captures the general trend of $\alpha(\epsilon)>\alpha(\sigma)$ which is frequently observed in sand (Yoshimine et al. 1998).
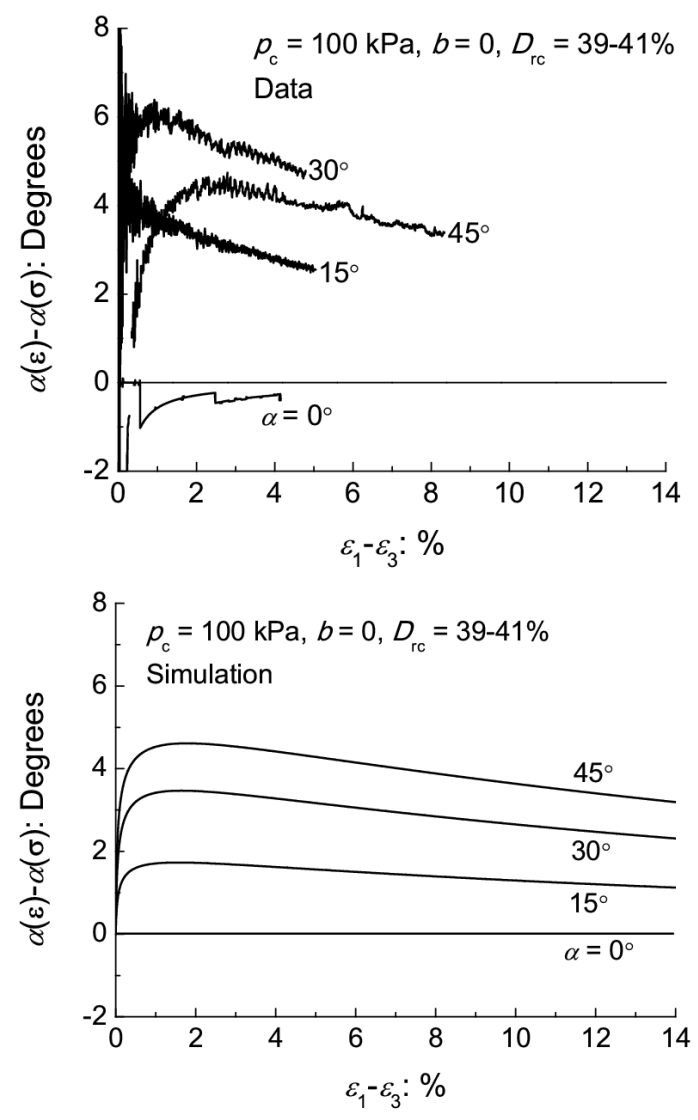

(a) $b=0$
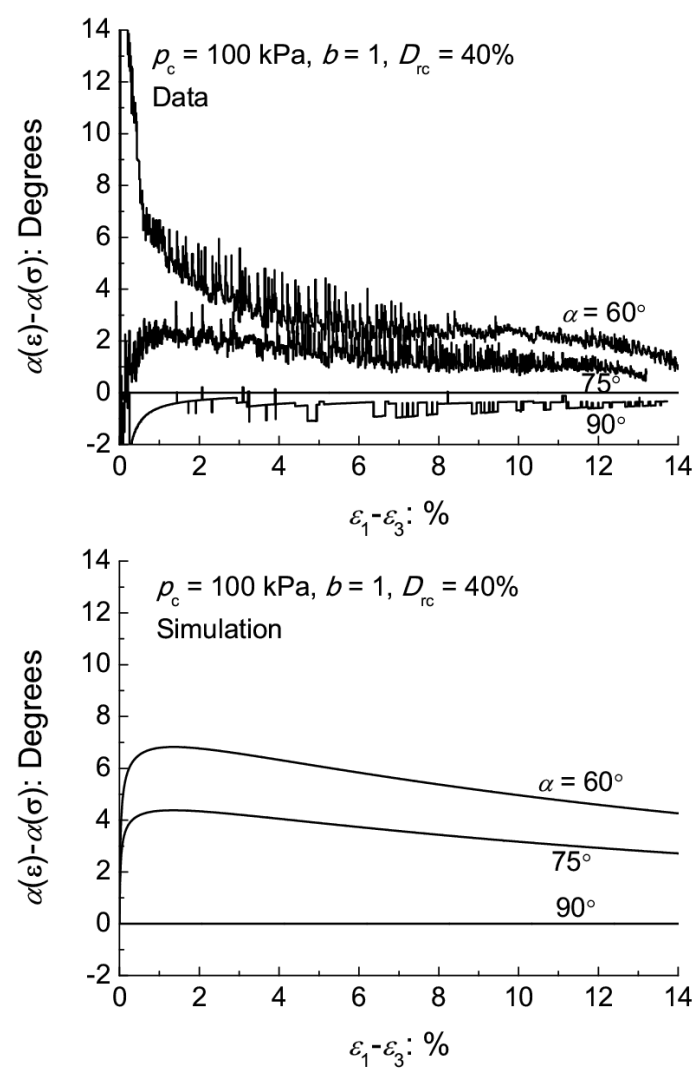

(b) $b=1$

Figure 2: Test data and model simulations for the noncoaxial behavior of Toyoura sand under undrained rotational shear at $b=1$ and $b=1(b$ : intermediate principal stress ratio). 


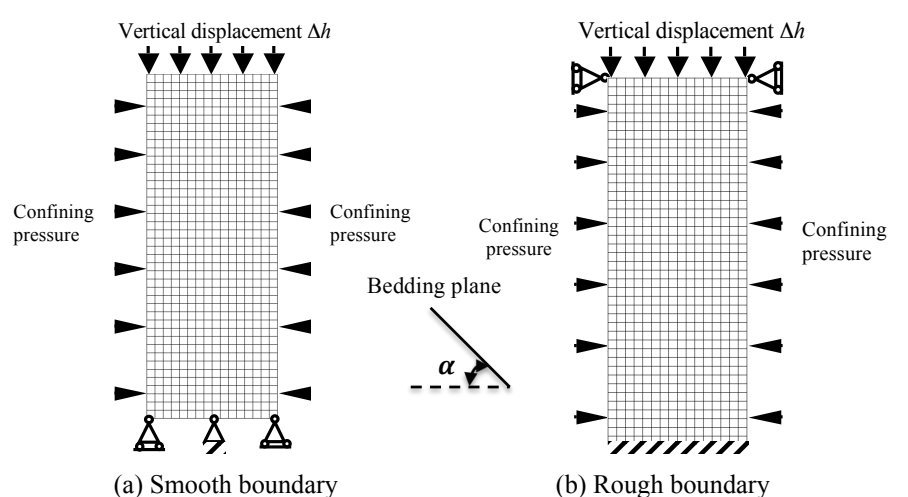

Figure 3: The finite element mesh used for simulation of biaxial compression of Toyoura sand with (a) smooth boundary and (b) rough boundary conditions.

\section{MODELLING THE SHEAR LOCALISATION IN SAND: THE ROLE OF FABRIC EVOLUTION}

Strain localization is frequently observed in sand and is considered an important precursor related to major geohazards such as landslides, debris flow and failures of relevant geo-structures. It is meanwhile recognised that the fabric in sand, representing an important internal structure, will constantly evolve during the loading course. There have been no previous studies investigating the correlation between strain localisation in sand and the fabric and its evolution which supposed to be extremely important towards better understanding the phenomenon of strain localisation. We employ the anisotropic sand model presented in previous sections in conjunction with finite element method to investigate the strain localisation in sand under plane strain compression, highlighting the role played by fabric evolution.

The proposed model has been successfully implemented in the finite element package ABAQUS through the user-material interface (UMAT) using an explicit integration method (Zhao et al. 2005). The implemented model is then used to investigate the strain localisation for Toyoura sand under plain strain compression treated experimentally by Tatsuoka et al. (1990). The dimension of the plane strain sand sample is $h \times w=10.5 \mathrm{~cm} \times 4 \mathrm{~cm}$. The domain is disretized by $42 \times 16$ four-nodes plane strain elements. A constant confining pressure is applied to the horizontal direction of the sample and an incremental vertical displacement is applied to the top end of the sample. Two types of boundary conditions are considered: smoothed boundary (left figure in Figure 3 ) and rough boundary (right figure in Figure 3 ). The bedding angle $\alpha$ is indicated in the figure. The initial void ratio distribution is assumed to be uniform throughout the sample. We take the case of bedding angle of $\alpha=45^{\circ}$ as a demonstrative example.

Gao and Zhao (2013) have found that for such a homogeneous sample with symmetric loading and boundary conditions, the anisotropic fabric can serve as a symmetry breaker triggering the strain localisa-

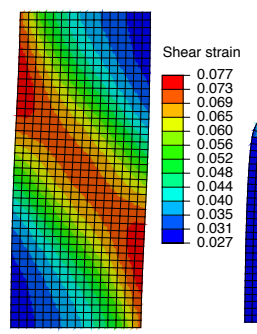

(a)

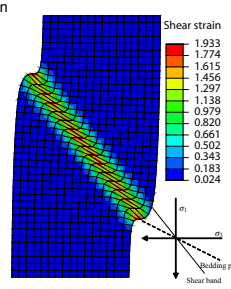

(b)

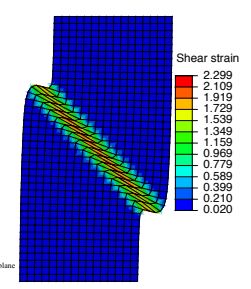

(c)
Figure 4: Distribution of shear strain for $\alpha=45^{\circ}$ with smooth boundary at (a) the peak stress state $\epsilon_{h}=4.2 \%$, (b) $\epsilon_{h}=12 \%$ with evolving fabric and (c) $\epsilon_{h}=12 \%$ with constant fabric $\left(\epsilon_{h}=\Delta h / h=\right.$ global vertical strain).

tion in the sample. Due to the non-coaxiality of the fabric with respect to the stress, a non-coaxial strain field is induced in each element with its major principal direction aligning with the an angle to the left of the vertical direction, which triggers the occurrence of localisation.

\subsection{Smooth boundary}

Figure 4 shows the development of shear band in sand sample with smooth boundary for the case of $\alpha=45^{\circ}$. Evidently a single asymmetric shear band is initialed around the peal global stress state and becomes intensely localised at the post-peak stage. Its orientation is close to the zero extension direction defined by (Roscoe 1958), which was termed by Tatsuoka et al. (1990) as Type-b shear band (aligning close to the bedding plane direction). When the fabric is fixed as a constant (by setting $k_{f}=0$ ), a similar pattern of shear band is found, however with more intensely concentrated shear strain within the band than considering fabric evolution. This indicates the sand may adapt its internal structure through the process of fabric evolution to alleviate the strain concentration.

\subsection{Rough boundary}

Figure 5 shows the evolution of shear bands for the case of rough boundary, $\alpha=45^{\circ}$ and considering fabric evolution. Strain localisation is found initiated before the peak global stress state. With rough boundary conditions, a shear band orientating to a Type-a pattern according to (Tatsuoka et al. 1990) (align close to the perpendicular direction of the bedding plane) develops substantially first, the failure of which is governed by the Coulomb's condition rather than the zero-extension mechanisms in the smooth boundary case. When $\epsilon_{h}$ reaches $5.8 \%$ (beyond the peak stress state), the second Type-b shear band starts to develop considerably while the first Type-a band continue to intensity, which leads to an cross shape double bands pattern at higher strain levels. The firstly occurring Type-b band remains the dominant one between the two. The overall shape of the two bands nevertheless appears to be symmetric. 


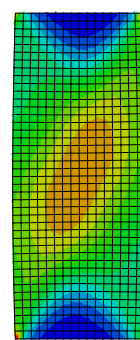

(a)

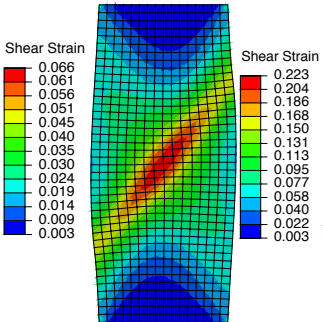

(b)

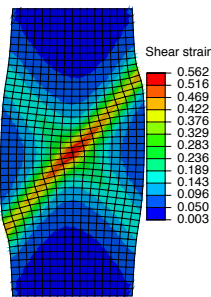

(c)

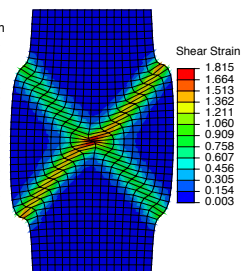

(d)
Figure 5: Evolution of shear band for sand sample under plane compression with $\alpha=45^{\circ}$ and rough boundary at four strain levels (considering fabric evolution): (a) $\epsilon_{h}=2.8 \%$; (b) $\epsilon_{h}=5.8 \%$; (c) $\epsilon_{h}=8.4 \%$ and (d) $\epsilon_{h}=15.5 \%$.

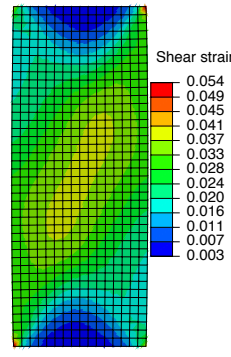

(a)

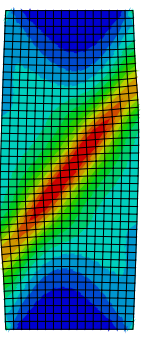

(b)

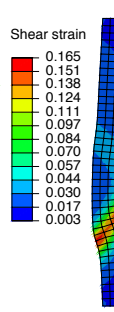

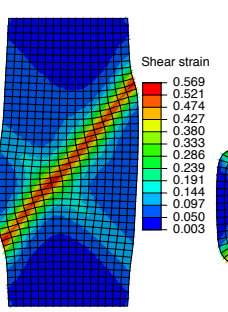

(c)

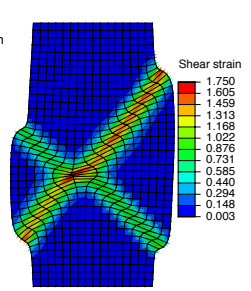

(d)
Figure 6: Evolution of shear band for sand sample under plane compression with $\alpha=45^{\circ}$ and rough boundary at four strain levels (the fabric is fixed as a constant): (a) $\epsilon_{h}=2.2 \%$; (b) $\epsilon_{h}=4.5 \%$; (c) $\epsilon_{h}=7.8 \%$ and $(\mathrm{d}) \epsilon_{h}=15.6 \%$.

To highlight the effect of fabric evolution, a comparison case with fixed fabric is presented in Figure 6. While the figure shows a similar occurrence sequence that a Type-a shear band occurs first followed by a Type-b shear band, the Type-a shear band appears to be more dominant than in the previous evolving fabric case which attracts the major localised strain in the post-peak development of localisation. The Type$\mathrm{b}$ band only has limited development due to the inability of self-adjusting through fabric evolution of the sample. The final resultant double bands pattern is hence a rather asymmetric cross-shape one.

\subsection{Two competing mechanisms for shear band}

Further investigations reveal that fabric evolution and the structural constraint imposed by the boundary conditions constitute two competing physical mechanisms governing the shear band formation. Fabric evolution may help a soil sample to adjust within itself to reduce non-coaxial response and render the sample to resist the external load more optimally to relieve strain localisation, while the structural constraint by the boundary tends to exert more biased stress on the sample which leads to intensified strain localisation on the existing shear band(s). Detailed examination of such quantities including the reaction force imposed on the sample by the top/bottom boundary ends and the evolution of anisotropic variable $A$ confirms the above explanations (Gao \& Zhao 2013).

\section{CONCLUSION}

A sand plasticity model accounting for fabric anisotropy and its evolution has been developed within the Anisotropic Critical State Theory (ACST). It was demonstrated the model possesses a natural non-coaxial flow rule with the inclusion of fabric anisotropy in the yield surface and could characterise the non-coaxial behaviour in sand reasonably well. The model has been further applied to the prediction of strain localisation in sand under plane strain compression wherein the important role of fabric evolution is highlighted.

\section{REFERENCES}

Been, K. \& Jefferies, M. G. (1985). A state parameter for sands. Géotechnique 35(2), 99-112.

Gao, Z. \& Zhao, J. (2013). Strain localization and fabric evolution in sand. International Journal of Solids and Structures 50, 3634-3648.

Gao, Z., Zhao, J., Li, X.-S. \& Dafalias, Y. F. (2014). A critical state sand plasticity model accounting for fabric evolution. International Journal for Numerical and Analytical Methods in Geomechanics 38, 370-390.

Guo, N. \& Zhao, J. (2013). The signature of shear-induced anisotropy in granular media. Computers and Geotechnics 47, 1-15.

Gutierrez, M. \& Ishihara, K. (2000). Non-coaxiality and energy dissipation in granular material. Soils and Foundations 40, 49-59.

Li, X. \& Dafalias, Y. F. (2012). Anisotropic critical state theory: the role of fabric. Journal of Engineering Mechanics 138(1), 263-275.

Roscoe, K. H. (1958). The influence of strains in soil mechanics. Géotechnique 20(2), 129-170.

Tatsuoka, F., Nakamura, S., Huang, C. \& Tani, K. (1990). Strength anisotropy and shear band direction in plane strain tests of sand. Soils Found. 30(1), 35-54.

Yoshimine, M., Ishihara, K. \& Vargas, W. (1998). Effects of principal stress direction and intermediate principal stress on undrained shear behavior of sand. Soils Found. 38(3), 179-188.

Zhao, J. \& Guo, N. (2013). Unique critical state characteristics in granular media considering fabric anisotropy. Géotechnique 63(8), 695-704.

Zhao, J., Sheng, D., Rouainia, M. \& Sloan, S. W. (2005). Explicit stress integration of complex soil models. International Journal for Numerical and Analytical Methods in Geomechanics 29, 1209-1229. 